\title{
L'autocitation vue par la théorie scandinave de la polyphonie linguistique (ScaPoLine)
}

\author{
Christian Manga (Université de Bergen, Norvège)
}

\begin{abstract}
The present work focuses on the discursive phenomenon of selfquotation, that we define with Rosier $(2008,118)$ as « the staging of one's own speech or opinion in the service of a specific argument on the mode of recall ( $I$ told you that) or performative mode (I tell you that).» Our aim is to study the particular functioning of self-quotation within the Scandinavian theory of linguistic polyphony (ScaPoLine). Depending on whether the self-quotation is in the mode of an earlier saying or a present one, we will postulate a typology of speakers: homogeneous speaker (self-quotation in the present) and heterogeneous speaker (self-quotation in the past).
\end{abstract}

Keywords: Self-quotation, ScaPoLine, polyphonic configuration, homogeneous speaker, heterogeneous speaker.

\section{Introduction}

La grammaire des formes de reprise à visée préférentiellement argumentative peut comprendre, entre autres, les reprises de type diaphonique, les anticipations et l'autocitation. Les deux premières, contrairement à la dernière, ont comme dénominateur commun, la reprise du discours d'autrui, antérieur ou à venir ; ce qui les range dans les tiroirs traditionnels du discours représenté ${ }^{1}$. Avec l'autocitation, nous sortons de la sémantique habituelle du discours représenté, pour y voir une autre variété orientée vers le dédoublement énonciatif du locuteur au service d'une argumentation spécifique, soit sur le mode du rappel (je vous ai dit que), soit sur le mode performatif (je vous dis que).

Ces clivages entre les formes de reprise laissent entrevoir les deux piliers du discours échoïque ou polyphonique: la polyphonie externe et la polyphonie interne. Le fonctionnement de la dernière au sein de la théorie scandinave de la polyphonie linguistique (ScaPoLine) fait l'essentiel de cette réflexion. Après un parcours sémantique, énonciatif et syntaxique de l'autocitation (1), nous en proposerons une structure polyphonique (2), qui débouchera sur une configuration polyphonique (3). Pour mener ce travail, notre corpus s'est constitué à partir des exemples tirés des discours du Président de la République du Cameroun.

\footnotetext{
${ }^{1}$ Nous préférons le terme discours représenté à celui de discours rapporté parce que le premier, proposé par Fairclough en 1998, fonctionne comme terme générique de toutes les catégories de discours rapportés. Étant donné que nous étudions, dans ce travail, l'autocitation, nous nous accommodons du terme discours représenté.
} 


\section{Sémantique, énonciation et syntaxe de l'autocitation ${ }^{2}$}

Dans ses travaux sur le discours rapporté / représenté, Marnette $(2006,38)$ se posait la question de savoir si le «discours rapporté » est exclusivement le discours d'autrui. L'auteure répondait elle-même de façon ironique : «oui, si je est un autre ». Cette réponse semble trancher un éventuel débat sur les formes du discours représenté, et les différentes catégories qui le composent. Étant donné, en effet, que je n'est pas un autre au sein de lui-même, et puisqu'il peut rapporter ses propres paroles, il s'ensuit que le discours représenté n'est pas seulement le discours d'autrui.

L'autocitation (désormais AC) en est une illustration. Dans un discours, le locuteur, selon sa visée de discours, peut structurer son argumentation sur plusieurs modes. Il peut décider de se mettre en scène dans sa propre énonciation en reprenant les discours ou les points de vue qu'il a développés précédemment dans le même énoncé ou dans un autre. Il peut par ailleurs s'auto-mettre en scène sur le mode performatif. C'est ainsi que pour Rosier (2008, 126) " l'autocitation (self-quotation) désigne la citation par soi d'un dire qui se présente dans le discours comme un dire antérieur [présent] ou supposé comme tel. »

L'AC est donc un discours égocentré, introverti, mis au service d'une argumentation spécifique. C'est dire que, du point de vue polyphonique, l'AC crée un dialogue ou une interaction entre le locuteur et lui-même, par effet de dédoublement où le « locuteur citant et le locuteur cité réfèrent au même sujet parlant. » (Rabatel 2006, 75)

Le phénomène d'AC peut transgresser les limites de la linguistique pour embrasser la littérature, le cadre social (Rosier, 2008). L'usage littéraire renvoie à une forme d'intertextualité où l'œuvre littéraire peut devenir son propre intertexte: un auteur qui se réfère à ses propres productions. Nous pouvons rencontrer ce phénomène chez un écrivain comme Camus, qui, dans la pièce de théâtre Le Malentendu (1944), se réfère à une partie du roman L'Étranger (1942) où il avait déjà souligné l'histoire d'un jeune, qui, ayant passé plusieurs années sans revoir sa mère et sa sœur cadette, retourne les voir sous anonymat dans un hôtel où elles travaillent. Les deux femmes formeront le dessein de l'assassiner pour le dépouiller par la suite. Sans le savoir, elles venaient de tuer le fils, le frère : le malentendu. Lorsque Camus en fait toute une pièce en 1944, il y a ainsi un discours échoïque autophonique (Perrin, 2006) en rapport avec la séquence de L'Étranger.

L'usage social de l'AC concerne un type de discours propre à une communauté de locuteurs particulière. En politique, par exemple, les acteurs produisent généralement des discours propres à leurs différents cadres idéologiques, par des formules telles que : chez nous le mot démocratie signifie... ou encore voilà comment nous disons les choses chez nous...Ainsi peut-on voir dans cet usage social du phénomène étudié, la position du locuteur comme celle de représentant d'un groupe, d'un milieu, d'une corporation dont il reproduit le discours en tant que marque déposée du groupe. Dans ce cas, le locuteur se fait le porte-parole de la corporation tout entière.

Dans ce travail, nous nous limitons à l'usage linguistique de l'AC, dont il faut décrire les variations lexicale, personnelle et temporelle du verbe, afin de mieux comprendre son fonctionnement au niveau polyphonique.

Relevons toutefois que la variation du verbe dans la syntaxe du discours égocentré n'est pas appréhendée unanimement chez les chercheurs qui s'intéressent à ce phénomène.

\footnotetext{
${ }^{2}$ Certains chercheurs parlent d'autophonie pour nommer le même phénomène. C'est le cas de l'école de Genève (Roulet et al. 2001), Perrin (2006), etc.
} 
Bien que partageant plusieurs points, certains travaux, comme ceux de Rabatel (2003), résument l'AC au dédoublement en un locuteur au passé.

Nous nous inscrivons dans un cadre d'analyse qui se réclame large et global de la réalité. Une telle approche nous met dans la ligne de description de Marnette (2006) et plus ou moins de Rosier (2008). Précisément, le travail de Marnette sera d'une importance capitale parce que cadrant avec nos objectifs. Pour l'auteure,

En d'autres mots, un locuteur tendra à citer ses propres pensées ici et maintenant (je pense que, je crois que) tout en présentant les opinions des autres en tant que paroles au présent ou au passé (X dit / a dit que), [...] D'une manière encore plus générale, le fait de prendre en compte dans mon analyse les expressions du type je dis et je pense m'a permis d'avoir une vue beaucoup plus complète (et complexe) des stratégies du discours rapporté, [donc de l'autocitation.] (Marnette 2006, 36-37)

Au regard de ce qui précède, l'on peut déduire qu'il y a une corrélation logique entre deux façons de citer: les expressions de type $X$ dit que / $X$ a dit que, et l'actualisation égocentrée de celles-ci à la première personne : je dis que / j'ai dit que. Les deux, selon Marnette, constituent des formes de « discours rapportés ».

Pour décrire le verbe dans l'AC, nous partons non seulement des universaux, c'est-àdire ce qui est commun, mais aussi de la variation performative du verbe introducteur.

\subsection{Variation lexicale du verbe}

Le verbe est un constituant important dans le fonctionnement des AC. Il peut jouer, outre la fonction d'introducteur, celle d'élément qui, par sa conjugaison, oriente la nature du discours autocité. Celui-ci est introduit en effet par une gamme de verbes de parole, de pensée, d'opinion et d'attitude propositionnelle. Vu le cadre restreint de cet article, nous n'allons pas les décrire tous. Mais pour les plus récurrents, à savoir les verbes de parole et d'opinion, ils servent, lorsqu'ils sont à la première personne, « soit à rapporter un discours ou une pensée du locuteur en tant qu'être du monde auquel l'énoncé réfère, soit à modaliser ce qui est exprimer par le locuteur en tant que responsable de l'énonciation effective. » (Perrin 2006, 42) Ici, la capacité des verbes de parole à modaliser l'expression du locuteur ne nous intéresse guère, c'est en revanche leur force à pouvoir insérer un discours dans un autre, à superposer deux situations d'énonciations qui importe.

Les verbes comme dire, affirmer, déclarer, répéter, annoncer, ajouter, poursuivre, reprendre, préciser, confirmer, apprendre, raconter, noter, conclure, révéler, dévoiler, expliquer... annoncent une phrase déclarative, qui, aux premières personnes du singulier et du pluriel, signalent une AC, dont la nature est tributaire de la variation temporelle du verbe.

\subsection{Variation temporelle du verbe}

Pour rester dans le canevas analytique de Marnette, il convient de considérer la variation temporelle du verbe introduisant l'AC. Les verba dicendi, tout comme les sentiendi, selon qu'ils sont sur le mode rappel ou sur le mode performatif, définissent le type d'AC. Sur le mode rappel, c'est-à-dire lorsque le dicendi est au passé, nous faisons face à un dédoublement en un locuteur antérieur (Ex : j'ai dit... / J'avais affirmé...). Sur le mode 
performatif, le dédoublement du locuteur se fait au présent, la double énonciation est ainsi ancrée dans la situation hic et nunc (Ex : je vous dis... / J'affirme).

Dans les sections consacrées à la configuration polyphonique des AC (Section 3, infra), nous verrons la différence à l'intérieur du locuteur, selon qu'on est au mode énonciatif antérieur ou au mode performatif. Avec le dédoublement au passé, le locuteur citant correspond au locuteur de l'énoncé, tandis que le locuteur cité renvoie au locuteur textuel (le locuteur à un autre moment de son histoire). Au mode performatif, le locuteur citant et le locuteur cité sont identiques. Cette distinction fait appel à la variation personnelle du verbe dans l'AC.

\subsection{Variation personnelle du verbe}

Nous avons décrit l'attitude du locuteur dans son auto-mise-en-scène-énonciative en rapport avec la variation des verba dicendi ou sentiendi. L'un des éléments importants dans la variation du verbe est, par ailleurs, la personne. La variation de celle-ci est un des traits de définition de l'AC. Il existe deux manières d'exprimer sa pensée ou de se mettre en scène, c'est le recours aux pronoms personnels je et nous (le nous pluriel ou le nous de modestie). La variation personnelle du verbe introducteur de l'AC oscille donc entre je et nous, qui, chez Benveniste (1966), font partie des vrais pronoms personnels (comme $t u$ ), car ils sont directement mis en contexte, et renvoient à leurs propres personnes, du point de vue pragmatique.

Toutefois, dans le langage des bébés, on peut avoir des AC à la troisième personne, il. Il s'agit ainsi d'un cas atypique et isolé. Tout compte fait, pour qu'il y ait autocitation « trois conditions, qui sont autant de caractéristiques linguistiques, sont nécessaires. » (Rabatel 2006, 71) : la présence d'un verbe de parole, de pensée ou d'opinion (dire, affirmer...) ; la variation temporelle du verbe (au passé ou au performatif) et la variation personnelle du verbe introducteur (je / nous).

Ce cadre sémantico-syntaxique de l'AC est un préalable propice à la compréhension de son fonctionnement dans la théorie scandinave de la polyphonie linguistique (ScaPoLine, Nølke et al. 2004).

\section{Structure et configuration polyphonique de l'AC dans la ScaPoLine}

La ScaPoLine est une théorie polyphonique développée par de chercheurs scandinaves (Nølke et al. 2004). Elle s'inspire des travaux de Ducrot (notamment 1984) sur la polyphonie linguistique. L'approche ducrotienne, essentiellement structuraliste, étudie la polyphonie au niveau de la langue, à partir des marqueurs signalant la superposition des points de vue mis en scène par le locuteur.

La ScaPoLine part des acquis de Ducrot, mais s'en distancie aussi sur un certain nombre de points :

[C]ontrairement à Ducrot, nous ne pourrons nous contenter d'examiner la polyphonie au niveau de la langue. Comme la plupart des autres linguistes qui se sont inspirés de Ducrot, nous aurons besoin de traiter la manifestation polyphonique au niveau de la parole également (Nølke et al. 2004, 19-20). 
La ScaPoLine entend fédérer les analyses linguistiques et les analyses textuelles. Cette orientation polyphonique permet de comprendre cette théorie comme une théorie sémantique, instructionnelle et énonciative. La dimension énonciative, qui manque au travail polyphonique de Ducrot, est capitale chez Nølke et ses collègues, car celle-ci est vectrice, non seulement d'une structure polyphonique, mais aussi d'une configuration. La structure polyphonique (structure-p) traite des phénomènes polyphoniques engendrés par la phrase, indépendamment de son contexte énonciatif. Il peut s'agir des phénomènes comme la négation polémique, la modalité épistémique, les connecteurs, etc. La structure-p donne donc des instructions nécessaires pour l'interprétation du sens des énoncés.

Quant à la configuration polyphonique, qui relève du niveau de la parole, elle engage le niveau énonciatif, le sens des énoncés. La configuration renferme les images des instances énonciatives, notamment celle du locuteur, qui construit la configuration polyphonique. Celleci, dans la ScaPoLine, comprend quatre éléments :

- Le locuteur en tant-que-constructeur (Abrégé LOC) assume la responsabilité de l'énonciation.

- Les points de vue (Abrégé pdv) sont des entités sémantiques porteuses d'une source qui est dite avoir le pdv. Les sources sont des variables ${ }^{3}$.

- Les êtres discursifs (Abrégé ê-d) sont des entités sémantiques susceptibles de saturer les sources.

- Les liens énonciatifs (liens) relient les ê-d aux pdv.

Nous n'allons pas développer, de fond en comble, chacun de ces quatre éléments de la configuration. En revanche, en raison de son importance dans ce travail, il est pertinent de clarifier le rôle et la complexité de LOC. Ce dernier, en tant que constructeur de l'appareil constitué de pdv, des ê-d et des liens, n'entre jamais en scène comme source d'un pdv. Une telle fonction est dévolue aux ê-d, qui correspondent à ce que Fløttum (2003) a appelé «personnes linguistiques », c'est-à-dire des entités de l'univers de discours pouvant établir des liens de référence linguistique.

Pour entrer en scène, LOC peut créer différentes images de lui-même : il peut être locuteur de l'énoncé actuel $\left(1_{0}\right)$, locuteur textuel $(\mathrm{L})$ et locuteur d'énoncé $\left(1_{\mathrm{t}}\right)$.

Le premier $\left(1_{0}\right)$ est responsable de l'énonciation hic et nunc, c'est lui qui réfère au pronom je / nous de la situation d'énonciation. Le locuteur textuel (L) a « tous les aspects d'une personne complète » (Nølke et al. 2004, 38); c'est une image de LOC à un autre moment de son histoire. La dernière image, le locuteur d'énoncé $\left(1_{t}\right)$ (Nølke 2009) correspond à l'image du locuteur à un moment $t(\neq 0)$ de l'énonciation, c'est la source d'un pdv que le locuteur avait à ce moment $t$.

Dans la description polyphonique, il sera pertinent de suivre ces différentes images de LOC des AC. Prenons l'exemple suivant pour illustrer les quatre composantes de la configuration polyphonique :

(1) L'islam n'est pas une menace.

Au niveau de la structure-p, la négation ne...pas donne des instructions relatives à la coexistence de deux points de vue dans cet énoncé : un point de vue positif antérieur, qui

\footnotetext{
${ }^{3}$ Les sources correspondent aux énonciateurs de Ducrot.
} 
aurait pensé que l'islam est une menace; point de vue que le locuteur réfute par le recours à la négation, à caractère polémique ici. La ScaPoLine configure cet exemple comme suit :

$$
\begin{aligned}
& \operatorname{pdv}_{1}:[\mathrm{X}](\text { VRAI (L'islam est une menace)) } \\
& \left.\operatorname{pdv}_{2}:\left[1_{0}\right]\left(\text { INJUSTIFIÉ } \operatorname{pdv}_{1}\right)\right)
\end{aligned}
$$

Le locuteur $1_{0}$ est responsable de l'énoncé à la forme négative $\left(\mathrm{pdv}_{2}\right)$. La source du point de vue positif $\left(\mathrm{pdv}_{1}\right)$ est inconnue $([\mathrm{X}])$. Chaque pdv exprime un jugement, qui peut correspondre au modus de l'énoncé (VRAI et INJUSTIFIÉ). Il existe un lien de responsabilité entre le locuteur de l'énoncé $\left(1_{0}\right)$ et $\operatorname{pdv}_{2}$; et un lien de non-responsabilité entre lui et $\operatorname{pdv}_{1}$.

À partir de ce préalable théorique, nous tentons d'étudier - et c'est le point essentiel de cet article - le fonctionnement de l'AC et, par implication, celui du discours représenté, au sein de la ScaPoLine. Nous tentons de postuler une structure-p et une configuration polyphonique. Le contexte énonciatif et morphosyntaxique de l'AC décrit ci-dessus donne à voir que ce phénomène, comme le discours représenté en général, peut être ancré dans la sémantique instructionnelle de la phrase. Au gré de la variation personnelle et lexicale du verbe, on arrive à la structure de l'AC suivante :

Je / nous - dire (que) - p, où le verbe dire tient lieu de représentant de tous les verbes pouvant introduire un discours représenté. Illustrons-le aves les exemples tires des discours du Président de la République du Cameroun, Paul Biya :

(2) A vous tous, qui nous avez soutenu si ardemment, et sans demander de contrepartie, je tiens à réaffirmer que vous n'êtes pas oubliés dans nos projets.

(3) J'ai eu l'occasion de dire que la nation, dans son ensemble, devait se mobiliser derrière son armée.

Ces occurrences d'AC sont tirées, respectivement, des discours du 06 octobre 1992 et du 31 décembre 2014. Le choix des discours n'est pas important dans cette étude, l'essentiel étant le phénomène que nous analysons.

En (2), la structure de l'AC est telle que le dicendi, réaffirmer, en emploi performatif actionné par la variation personnelle du verbe en je, ancre le dédoublement énonciatif dans la situation hic et nunc. Relevons que malgré le statut d'infinitif de réaffirmer, sa variation temporelle est tributaire de celle du verbe qui le précède (tiens). Ce dernier lui confère ainsi toutes ses propriétés morphosyntaxiques. En reconstruisant, (2) pourrait prendre cette forme :

(2) ' je réaffirme que vous n'êtes pas oubliés dans nos projets.

Cette occurrence répond à la structure Je / nous - dire (que) - p de l'AC. Nous obtenons la configuration polyphonique ci-dessous :

$$
\begin{aligned}
& \left.\operatorname{pdv}_{1}:\left[1_{0}\right](\text { VRAI (je réaffirme } p)\right) \\
& \operatorname{pdv}_{2}:\left[1_{0}\right] \text { (VRAI (Vous n'êtes pas oubliés)) }
\end{aligned}
$$

Soulignons que notre intérêt n'est pas d'analyser la négation polémique incluse dans le

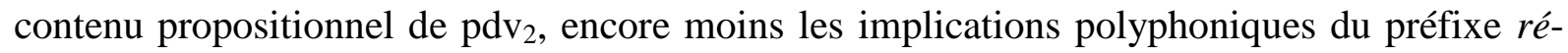
(de réaffirmer) ; seule le fonctionnement de l'AC nous intéresse dans cette étude. 
Prenons (3) (J'ai eu l'occasion de dire que la nation, dans son ensemble, devait se mobiliser derrière son armée), qui peut être aussi reconstitué comme suit :

(3) ' J'ai dit que la nation, dans son ensemble, devait se mobiliser derrière son armée.

Nous avons un verba dicendi au passé, et sa variation personnelle à la première personne. Nous pouvons configurer cette occurrence comme suit :

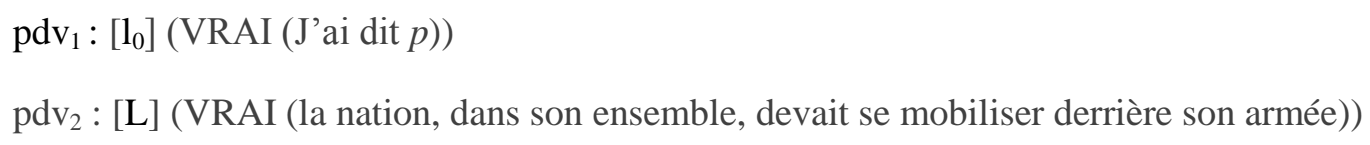

Lorsque nous comparons les configurations polyphoniques de (2) et (3), nous nous rendons à l'évidence que la nature du locuteur dans l'AC varie en fonction du type de dédoublement énonciatif. Dans un dédoublement au passé (comme dans l'exemple 3), nous avons le locuteur de l'énoncé, source de $\mathrm{pdv}_{1}$, et une de ses images, en l'occurrence le locuteur textuelle (L), qui est le locuteur à un autre moment de son histoire (cf. Nølke et al. 2004), source de $\operatorname{pdv}_{2}$. Ce locuteur (L) est lié à une situation d'énonciation autre que celle au présent.

Lorsque l'AC s'opère sur le mode performatif, le locuteur de l'énoncé $\left(1_{0}\right)$ est responsable des deux actes d'énonciation: il est à la fois la source de $\operatorname{pdv}_{1}$ et de $\operatorname{pdv}_{2}$ (exemple 2).

\section{Conclusion}

Notre réflexion a porté sur le fonctionnement de l'AC dans la théorie scandinave de la polyphonie linguistique. Il s'ensuit que cette variante du discours représenté repose sur une structure morphosyntaxique (structure-p) dont la configuration polyphonique détermine la variation du locuteur. En fonction du type de dédoublement énonciatif dans l'AC, le locuteur peut passer de l'aspect homogène à l'aspect hétérogène. Image homogène du locuteur lorsque l'AC s'opère sur le mode performatif, où $l_{0}$ est à la fois source de $\mathrm{pdv}_{1}$ et $\operatorname{de} \mathrm{pdv}_{2}$. Nous avons une image hétérogène du locuteur lorsque le dédoublement énonciatif se fait au passé ; dans ce cas le locuteur de l'énoncé $\left(l_{0}\right)$ et le locuteur textuel $(\mathrm{L})$ sont respectivement source de $\mathrm{pdv}_{1}$

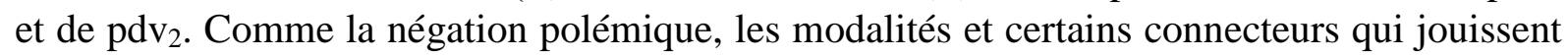
d'un modèle de configuration polyphonique au sein de la ScaPoLine, nous avons parallèlement décrit le fonctionnement polyphonique de l'AC.

\section{Bibliographie}

Bakhtine, Mikhaïl. 1970 [1963]. Problèmes de la poétique de Dostoïevski. Lausanne : L’âge d'homme.

Benveniste, Emile. 1966. Problèmes de linguistique générale (Tome1). Paris : Gallimard.

Benveniste, Emile. 1974. Problèmes de linguistique générale (Tome2). Paris : Gallimard.

Camus, Albert. 1942. L'Étranger. Paris : Gallimard.

Camus, Albert. 1944. Le Malentendu. Paris : Gallimard. 
Ducrot, Oswald. 1972. Dire et ne pas dire: principes de sémantique linguistique. Paris : Hermann.

Ducrot, Oswald. 1984. Le dire et le dit. Paris : Editions de Minuit.

Fløttum, Kjersti. 1999. Polyphonie et typologie : quelques questions. Bergen : Université de Bergen, Institut d'études romanes : 81-96.

Fløttum, Kjersti. 2002. «Polyphonie et typologie revisitées » Polyphonie - linguistique et littéraire V, Roskilde. Samfundslittératur Roskilde : 1-38.

Fløttum, Kjersti. 2003. «Polyphonie dans les textes scientifiques. Étude de deux cas français »Polyphonie - linguistique et littéraire VII, Roskilde. Samfundslittératur Roskilde : 111-129.

Marnette, Sophie. 2006. « Je vous dis que l'autocitation c'est du discours rapporté. » Travaux de linguistique 1 , no. $52: 25-40$.

Marnette, Sophie, Muñoz, López Manuel Juan, Rosier, Laurence. 2006. « Autocitation et genres de discours, quelques balises » Travaux de linguistique 1, no. 52: 7-23.

Nølke, Henning. 2009. « Types d'êtres discursifs dans la ScaPoLine » Langue française 4, no. $164: 81-96$.

Nølke, Henning, Fløttum, Kjersti, Norén, Coco. 2004. La Scapoline: la théorie scandinave de la polyphonie linguistique. Paris : Editions kimé.

Perrin, Laurent. 2006. « Autophonie et formules modales. Verbes de parole et d'opinion à la première personne » Travaux de linguistique 1 , no. $52: 41-55$.

Rabatel, Alain. 2003. « Les verbes de perception en contexte d'effacement énonciatif : du point de vue représenté aux discours représentés » Travaux de linguistique 46-1 : 4988.

Rabatel, Alain. 2006. «Les auto-citations et leurs reformulations : des surassertions

surénoncées ou sousénoncées. » Travaux de linguistique 1, no. 52 : 71-84.

Rosier, Laurence. 1999. Le discours rapporté; Histoire, théories, pratiques. Paris / Bruxelles : DeBoeck-Duculot.

Rosier, Laurence. 2008. Le discours rapporté en français. Paris : Editions Ophrys.

Vincent, Diane, Dubois, Sylvie. 1997. Le discours rapporté au quotidien. Québec: Nuit Blanche éditeur. 